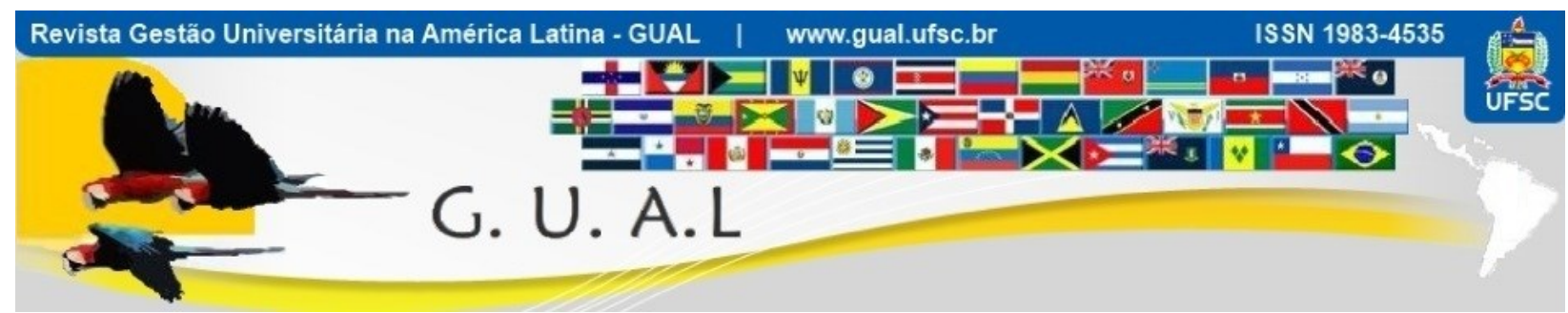

DOI: http://dx.doi.org/10.5007/1983-4535.2015v8n3p1

\title{
LEVANTAMENTO DE REQUISITOS PARA A PROPOSTA DE UM APLICATIVO PARA ACOMPANHAMENTO DOS QUESITOS DO SISTEMA DE AVALIAÇÃO DA CAPES
}

\section{REQUIREMENTS GATHERING FOR THE PROPOSAL OF AN APPLICATION FOR MONITORING THE ASPECTS OF THE EVALUATION SYSTEM OF CAPES}

Emerson Antonio Maccari, Doutor Universidade Nove de Julho - UNINOVE maccari@,uninove.br

José Carmino Gomes Junior, Mestre Universidade Nove de Julho - UNINOVE mat.jose.carmino@gmail.com

Claudia Terezinha Kniess, Doutora Universidade Nove de Julho - UNINOVE ctkniess@uninove.br

Cesar Augusto Biancolino, Doutor Universidade Nove de Julho - UNINOVE biancolino@gmail.com

Recebido em 01/outubro/2014

Aprovado em 24/julho/2015

Sistema de Avaliação: Double Blind Review

Esta obra está sob uma Licença Creative Commons Atribuição-Uso. 


\title{
RESUMO
}

A gestão dos Programas de Pós-Graduação Stricto Sensu com vistas ao atendimento aos critérios da CAPES tem se mostrado como uma atividade complexa, pois se baseia em parâmetros dinâmicos e heterogêneos ao longo do tempo. $\mathrm{O}$ artigo teve como base o método da pesquisa-ação que levantou os requisitos para o desenvolvimento de um sistema de acompanhamento dos quesitos do sistema de avaliação da CAPES com vistas a auxiliar a gestão estratégica dos Programas Stricto Sensu. Identificou-se que este aplicativo pode ser incorporado à rotina dos coordenadores dos Programas visando dar suporte ao planejamento estratégico em busca do seu aperfeiçoamento. Como resultado, vislumbra-se uma melhor gestão dos indicadores de produção científica e de formação dos alunos, critérios estes fundamentais para que o programa possa cumprir sua missão perante a sociedade.

Palavras-chave: Gestão de Programas de Pós-Graduação. CAPES. Tecnologia da Informação. Gestão Estratégica.

\begin{abstract}
The management of Graduate Programs are doing to meet the criteria of CAPES. This task has proven to be a complex activity because it is based on dynamic and heterogeneous over time parameters. The article was based on the method of action research that raised the requirements for developing a monitoring system of the requirements of the assessment system of CAPES with a view to assisting the strategic management of the graduate studies program. It was identified that this application can be incorporated into routine coordinators of programs aiming to support strategic planning in pursuit of improvement. As a result, one can glimpse a better management of scientific production indicators and training of students, these fundamental criteria for the program to fulfill its mission to society.
\end{abstract}

Key words: Graduate Program Management. CAPES. Information Technology. Strategic Management. 


\section{INTRODUÇÃO}

Em seus anos de existência, a Pós-Graduação brasileira vem se expandindo de forma significativa, firmando-se e alcançando credibilidade internacional com altos padrões de qualidade em várias áreas do conhecimento. Neste sentido, pode-se concluir que a PósGraduação vem ao longo destes anos contribuindo sobremaneira para o desenvolvimento do país (KUENZER; MORAES, 2005, MACCARI et al. 2009).

Criada na década de 1950, com a finalidade de expandir e consolidar a Pós-Graduação Stricto Sensu em todos os Estados da Federação, a Coordenação de Aperfeiçoamento de Pessoal de Nível Superior (CAPES) contribui para que os cursos de Pós-Graduação funcionem de modo integrado e consistente para o desenvolvimento de conhecimento tecnológico e científico que a sociedade necessita (CAPES 2012a). Neste sentido, a CAPES tem contribuído para o desenvolvimento em quantidade e qualidade da Pós-Graduação Stricto Sensu no Brasil. Isto é notado pelo expressivo aumento dos cursos de mestrado e doutorado nos últimos anos e pela evolução nos indicadores de qualidade do Sistema de Avaliação da Pós-Graduação utilizado pela CAPES (MACCARI et al., 2007). A existência de quesitos para medir a qualidade dos Programas Pós-Graduação Stricto Sensu faz com que se exija cada vez mais rigor nos procedimentos de avaliação da qualidade, estimulando o aperfeiçoamento contínuo do sistema (OLIVEIRA et al., 2008).

Para confirmar sua importância e magnitude, em agosto de 2014 a Pós-Graduação Stricto Sensu brasileira estava constituída por 3.805 Programas, sendo 1.283 Programas com o mestrado acadêmico, 579 com mestrado profissional, 62 só com doutorado e 1.881 Programas com mestrado e doutorado acadêmico (CAPES, 2014a).

A avaliação dos Programas de Pós-Graduação compreende a realização do acompanhamento anual e a avaliação trienal do desempenho de todos os Programas e cursos que integram o Sistema Nacional de Pós-Graduação (SNPG). Os resultados desse processo são expressos pela atribuição de uma nota na escala de "1" a "7", fundamentado pela deliberação do Conselho Nacional de Educação (CNE) e o Ministério da Educação e Cultura (MEC) sobre quais cursos obterão a renovação de "reconhecimento", a vigorar no triênio subsequente (CAPES, 2012b).

De acordo com Balbachevsky (2005), o processo de avaliação da CAPES foi aceito como a mais importante referência de qualidade para os Programas de Pós-Graduação no Brasil. A avaliação permitiu que se criasse uma conexão entre desempenho e sucesso: quanto 
melhor a avaliação alcançada pelo Programa, maiores eram suas chances e as de seus pesquisadores de alcançar apoio tanto em bolsas de estudo como em recursos para pesquisa e infraestrutura.

Atualmente enfrenta-se um intenso debate quanto ao papel e às finalidades da PósGraduação no Brasil e da qualidade de suas propostas acadêmicas. Num momento em que existem demandas por Programas de Pós-Graduação que respondam à grande variedade de desafios sociais, tecnológicos, políticos e ecológicos, é de grande importância refletir sobre as condições atuais e o futuro desejável para estes Programas (MACCARI, et. al., 2013).

Para Maccari (2008) os Programas de Pós-Graduação Stricto Sensu podem melhorar seu desempenho gerencial se considerarem o sistema de avaliação da CAPES para estabelecer um planejamento estratégico e metas associadas. Esta avaliação também serve como direcionador às estratégias adotadas pelas coordenações dos Programas. Estudar e compreender como os Programas ajustam suas estruturas para se adaptarem ao processo de avaliação torna-se prioritário para o fortalecimento do sistema como um todo.

Em relação aos sistemas de avaliação da Pós-Graduação em outros países, estudos realizados por Maccari et. al. (2014), com vistas a comparar o sistema de avaliação do Brasil (CAPES) e dos Estados Unidos (AACSB), descrevem que o sistema de avaliação realizado no Brasil tem foco no resultado, onde os critérios se pautam em indicadores na sua maioria quantitativos, orientados no produto. Já o sistema de avaliação norte-americano é centrado nos processos de ensino e aprendizagem, o que coloca os quesitos e indicadores em um caráter predominantemente qualitativo, com ênfase no processo.

Partindo do contexto da importância da atuação da CAPES e de seu sistema de avaliação para a continuidade e fortalecimento da Pós-Graduação no Brasil que, por consequência, contribui para o desenvolvimento de conhecimento científico e tecnológico do país, identifica-se a necessidade de investigar, de forma aprofundada como os Programas de Pós-Graduação se adaptam a esse sistema. Assim, tem-se a possibilidade de identificar estratégias que contribuem para o desenvolvimento e aprimoramento destes Programas.

Por meio da análise dos resultados das avaliações disponíveis no sítio da CAPES é possível acompanhar a evolução das notas dos Programas ao longo dos triênios. Ao analisar os demais dados disponíveis é possível identificar os efeitos das implantações de estratégias nos Programas, que contribuíram, efetivamente, para o desenvolvimento e aprimoramento e 
consequentemente para atender aos padrões do sistema de avaliação (MACCARI, et. al., 2013).

Neste contexto, um estudo sobre as estratégias adotadas e os recursos alocados pelos Programas de Pós-Graduação Stricto Sensu da área de Administração, Ciências Contábeis e Turismo (Martins et. al., 2013), realizada em oito Programas avaliados pela CAPES no período de 2001 a 2009, constatou que os Programas apresentaram estratégias deliberadas sistematicamente ao longo dos anos, tendo como alvo a melhoria de seus desempenhos no sistema de avaliação da CAPES. Como consequência, os Programas melhoraram suas estruturas de pesquisa e disponibilizaram recursos a seus pesquisadores para que eles ampliassem o foco nas atividades de pesquisas e orientações. Observou-se também indícios do uso do Resource Based - View - RBV como instrumento auxiliador na escolha das estratégias adotadas e na alocação dos recursos.

Esta pesquisa concentra-se no estudo do sistema de avaliação da CAPES de Programas de Pós-graduação Stricto Sensu no Brasil e como os Programas podem melhorar seu desempenho gerencial se considerarem este sistema de avaliação para estabelecer um planejamento estratégico e metas associadas. Sabe-se que os resultados da avaliação servem de base para a formulação de políticas para a área de Pós-Graduação, bem como para o dimensionamento das ações de fomento - bolsas de estudo, auxílios, apoios -, estabelecendo, ainda, critérios para o reconhecimento pelo Ministério da Educação dos cursos de Mestrado e Doutorado novos e em funcionamento no Brasil (CAPES 2012b, NEVES et. al., 2006).

Nesse sentido, a gestão estratégica da Tecnologia da Informação (TI) pode exercer um importante papel de apoio à estratégia e a gestão dos Programas de Pós-Graduação Stricto Sensu. No ambiente acadêmico, a TI tem uma aplicação significativa em função da natureza do trabalho científico, direcionado, primordialmente, para o intercâmbio de informações e a troca de experiências entre os pesquisadores (FLORES, 1999). Assim, as ferramentas de TI podem auxiliar na coleta e consolidação das informações dos Programas para um posterior acompanhamento sistemático de sua evolução.

O foco do estudo são os Programas da área de Administração, Ciências Contábeis e Turismo. Esta é uma das maiores áreas do sistema de avaliação da CAPES, apresentando nos últimos anos acentuado crescimento. Segundo o relatório da última avaliação trienal da CAPES (2010-2012) - Trienal 2013 - a área de Administração, Ciências Contábeis e Turismo contava, no ano de 2012, com 121 Programas de Pós-Graduação. Este numero representa os 
Programas recomendados pela CAPES e implantados até o ano de 2012, ou seja, desconsidera os Programas aprovados em 2012. Estes Programas estavam distribuidos da seguinte maneira: 38 mestrados e doutorados integrados, 39 mestrados, 2 doutorados e 43 mestrados profissionais (CAPES, 2013b).

Desta forma, o presente estudo pretende responder à seguinte questão de pesquisa: Como determinar os requisitos necessários para o desenvolvimento de um aplicativo de acompanhamento dos quesitos do sistema de avaliação da CAPES para os Programas de PósGraduação da área de Administração, Ciências Contábeis e Turismo? Nestes termos, o objetivo do trabalho é propor um aplicativo para o acompanhamento, pelos Programas de PósGraduação da área de Administração, Ciências Contábeis e Turismo, dos quesitos do sistema de avaliação da CAPES ao longo do tempo.

O aplicativo de acompanhamento dos quesitos do sistema de avaliação da CAPES contribuirá para a realização da análise sistemática e dinâmica das métricas associadas, sendo possível visualizar os quesitos nos quais Programa precisa incrementar, de modo a contribuir para uma boa avaliação. $\mathrm{O}$ aplicativo também permitirá fazer simulações simultâneas quanto as métricas associadas aos quesitos de avaliação.

O trabalho está estruturado em cinco seções, incluindo a parte introdutória, na qual foi apresentada sua contextualização, a problemática de pesquisa e os objetivos. Na seção dois são apresentados os polos teóricos da pesquisa. A seção três traz a metodologia da pesquisa. A seção quatro reúne os resultados e discussões da pesquisa e, por fim, na última seção, as considerações finais e recomendações.

\section{REVISÃO TEÓRICA}

Neste tópico é apresentada a fundamentação teórica do estudo. Trata-se da revisão da literatura realizada que serve para o suporte conceitual ao tema abordado. Para tanto, esta revisão fundamenta-se nos seguintes pilares: sistema de Pós-Graduação no Brasil, gestão estratégica de TI e a gestão de projetos.

\subsection{SISTEMA DE PÓS-GRADUAÇÃO NO BRASIL}

Como marco inicial da consolidação da Pós-Graduação no Brasil, destaca-se no ano de 1965, o parecer 977, conhecido como "Parecer Sucupira" do atual CNE que promove a implantação dos cursos de Pós-Graduação, Mestrado e Doutorado. O documento caracteriza o 


\section{LEVANTAMENTO DE REQUISITOS PARA A PROPOSTA DE UM APLICATIVO PARA

Mestrado como etapa preliminar na obtenção do grau de Doutor, ou ainda, como grau terminal para os que não pretendam seguir a carreira acadêmica. São também estabelecidas duas finalidades para os cursos de Stricto Sensu: a) Formação em caráter terminal de profissionais para o mercado de trabalho e b) Formação de pesquisadores para a vida acadêmica (BOMENY, 2001).

O crescimento da Pós-Graduação Stricto Sensu no Brasil é notório, podendo ser observado pelos resultados publicados no Plano Nacional de Pós-Graduação (PNPG) 20112020, o qual relata que o Sistema Nacional de Pós-Graduação (SNPG) evoluiu em uma curva ascendente no que diz respeito ao número de cursos recomendados pela CAPES e habilitados ao funcionamento ao final do ano base. O relatório traz as taxas de crescimento comparativamente entre os anos de 1976 a 2009 e entre 2004 e 2009, apresentadas na Tabela 1.

Tabela 1 Número de cursos de Pós-Graduação em atividade no Brasil.

\begin{tabular}{clllll} 
Nível & \multicolumn{2}{c}{1976} & \multicolumn{2}{c}{2004} & \multicolumn{2}{c}{2014} & \multicolumn{2}{c}{ Crescimento (\%) } \\
& & & & $2014 / 1976$ & \multicolumn{1}{c}{$2014 / 2004$} \\
\hline Mestrado & 518 & 1.793 & 3.158 & $509,65 \%$ & $76,12 \%$ \\
Mestrado Profissional & 0 & 119 & 573 & - & $381,51 \%$ \\
Doutorado & 181 & 1.058 & 1.943 & $973,48 \%$ & $83,64 \%$ \\
Total & 699 & 2.970 & 5.674 & $711,73 \%$ & $91,04 \%$ \\
\hline
\end{tabular}

Fonte: (CAPES, 2014b); (Brasil. MEC/CAPES, 2010)

Segundo o PNPG (2011-2020) o SNPG deverá ser fortemente expandido para atender às necessidades do país e atingir metas compatíveis com as nações avançadas. Neste sentido, suas metas serão: (a) aumento do número de doutores por mil habitantes, na faixa etária de 25 aos 64 anos, dos atuais 1,4 para 2,8 em 2020; (b) titulação anual de 19.000 doutores, 57.000 mestres e 6.000 mestres profissionais, em 2020.

Para cumprir o seu papel, a CAPES vem desenvolvendo o seu sistema de avaliação da Pós-Graduação ao longo de mais de 30 anos, entendendo que seja necessária a especificação, com nitidez, da relação que se estabelece entre critérios científicos e objetivos sociais, bem como a forma de elaboração de conceitos, seu domínio de validade e que conclusões podem ser realmente sustentadas pela metodologia empregada. Nessa linha, Gatti et. al. (2003) afirmam que qualquer processo de avaliação só tem características científicas e sentido social quando se estão bem definidos o contexto de referência e os objetivos.

Os resultados da busca pelo aperfeiçoamento do sistema de avaliação ficaram mais evidentes a partir do ano de 1998, que de acordo com Sampaio (2000), é apontado como um 


\section{LEVANTAMENTO DE REQUISITOS PARA A PROPOSTA DE UM APLICATIVO PARA

marco importante no mecanismo de avaliação dos Programas de Pós-Graduação da CAPES. Neste ano, ocorreu a implantação de um sistema padronizado de notas, critérios de qualidade da produção científica e de formação de alunos, considerados fatores primordiais a melhoria do desempenho dos cursos, em substituição dos conceitos adotados até então.

Inserido neste contexto, o sistema de avaliação da CAPES segue uma série de princípios e objetivos que buscam fundamentar as ações do processo avaliativo, que são: (a) regularidade do processo de acompanhamento e avaliação; (b) avaliação a cargo dos pares, estes escolhidos criteriosamente; (c) busca continua da elevação dos padrões de qualidade; (d) constante ajustamento dos critérios e indicadores com base nos novos patamares alcançados pela pesquisa científica e tecnológica (CAPES, 2012a).

Os resultados dos acompanhamentos são expressos pela atribuição de conceitos na escala de "1" a "7" que fundamentam a deliberação CNE/MEC sobre quais cursos obterão a renovação de "reconhecimento", a vigorar no triênio subsequente (CAPES, 2011c). O curso passa a integrar o sistema nacional de Pós-Graduação quando obtém nota mínima igual a 3. A avaliação dos Programas é estruturada e elaborada levando-se em conta os seguintes quesitos: I - Proposta do Programa; II - Corpo docente; III - Corpo discente; IV - Produção Intelectual; V - Inserção Social.

Para atingir os objetivos propostos, a CAPES utiliza algumas ferramentas e instrumentos, entre eles o sistema de avaliação por fichas, o Sistema de Indicadores de Resultados (SIR), cadernos de indicadores de resultados e planilhas específicas. O sistema de avaliação por fichas é o instrumento para o registro das avaliações de cada um dos Programas de Pós-Graduação CAPES (2012d). Existem fichas específicas para os Programas acadêmicos e para mestrados profissionais, sendo que ambas têm como objetivo garantir uma base uniforme e padronizada do processo de avaliação, levando em consideração as especificidades de cada área, como também a ampliação da integração no âmbito de sua respectiva grande área e no contexto geral.

De acordo com Maccari (2008), o SIR contribui para sistematizar e consolidar os indicadores da qualidade dos resultados produzidos pelos Programas de Pós-Graduação, sendo utilizado para comparar desempenhos e dirimir dúvidas das instâncias envolvidas na condução da avaliação. Já as planilhas específicas, são elaboradas para complementar as informações fornecidas pelos cadernos de indicadores. As Comissões de Área solicitam junto à CAPES a elaboração de relatórios específicos com dados e indicadores de desempenho dos 
Programas a serem avaliados, proporcionando realizar cruzamentos de dados que auxiliem na análise dos Programas de sua área.

\subsection{GESTÃO ESTRATÉGICA DE TI}

Tachizawa e Andrade (2003) afirmam que o papel da tecnologia da informação (TI) no contexto das organizações tem-se direcionado para o uso estratégico, sendo associado à agregação de valor e ao incremento da produtividade. Desta forma tem-se conduzido a uma priorização de investimentos em TI tais como: comércio eletrônico, software de gestão integrada, data warehousing, infraestrutura de hardware e software.

Para Lederer e Sethi (1988), o planejamento de sistemas de informação e da TI é um processo ativo, sistêmico, coletivo, continuo e, sobretudo participativo, objetivando assim a formalização da estrutura das informações e dos sistemas de informação necessários para uma tomada de decisão melhorada apoiando então a gestão da organização.

Noutro aspecto tem-se que o planejamento de sistemas de informação e da TI, apesar de tratar de recursos técnicos, é distinto do antigo Plano Diretor de Informática que está sobre o plano de informática e seus respectivos recursos tecnológicos (REZENDE, 1999). Lederer e Mahaney (1996) afirmam que o planejamento de sistemas de informação e da TI é um constante processo de identificação de software, de hardware e principalmente de banco de dados que dão suporte as regras do negócio alinhando-se assim as expectativas da organização.

Desta forma, a relevância do planejamento está depositada no alinhamento entre os sistemas de informação, sistemas de conhecimento e a TI com as metas dos negócios da organização, sendo que os principais objetivos são: a exploração das tecnologias de informação para obtenção de vantagem competitiva; direcionamento dos recursos para uma efetiva gestão; desenvolvimento constante de políticas e arquiteturas de tecnologia; construção constante de um ambiente informacional que favoreça a geração de estratégias organizacionais (PARSONS, 1983; EARL, 1993; DAVENPORT; PRUSAK, 1998; TURBAN; RAINER, JR; POTTER 2007).

Este alinhamento implica em uma melhor tomada de decisão e está apoiada na premissa de que é difícil tomar decisões sem informações relevantes e validas. Isto torna as informações necessárias para cada uma das etapas da atividade do processo decisório (TURBAN; RAINER, JR; POTTER 2007). 
Nesse contexto, este estudo busca relacionar a gestão estratégica de TI e a gestão de projetos com o sistema de avaliação da CAPES de Programas de Pós-Graduação Stricto Sensu, no sentido de desenvolver um aplicativo para o acompanhamento dos quesitos de avaliação, servido como uma ferramenta de apoio para o planejamento estratégico dos Programas e processo de tomada de decisão.

\subsection{GESTÃO DE PROJETOS DE TI}

A adoção de técnicas de gerenciamento de projeto foi incorporada à engenharia de software devido ao grande número de fracassos em grandes projetos nas décadas de 60 e 70 . Entretanto, Sommerville (2008) o distingue de outros tipos de projetos de engenharia, postulando as seguintes dificuldades como justificativas para atrasos em cronogramas e não cumprimento do orçamento: (a) o produto é intangível; (b) não existe um processo de software padrão; (c) grandes projetos de software são, frequentemente, projetos únicos.

Kerzner (2000) destaca que equipes de projeto altamente qualificadas enxergam o gerenciamento de requisitos e do escopo do projeto, como os elementos mais críticos da gestão de projetos. O projeto e seus requisitos têm início com as necessidades expressas e terminam apenas quando as necessidades são satisfeitas evidenciado pela validação do usuário o que o torna bem sucedido.

Em algumas organizações, devido às suas particularidades inerentes, há um esforço no sentido de desenvolvimento de abordagens customizadas em gerenciamento de projetos. Tal é o caso da indústria de softwares, que foi objeto de estudos por parte de diversos autores, entre eles: Bhoem (1991), Boehm e DeMarco (1997), Fairley (1994) e Presman (2006).

Bhoem (1991), Boehm e DeMarco (1997) ressaltam a dificuldade em se definir o escopo do software na fase de concepção e elaboração do projeto, por meio de técnicas de levantamento de requisitos junto ao cliente. De acordo com Boehm (1988), a fase inicial de documentação das necessidades do cliente não deve ser um processo "desgastante" para se atingir seus objetivos, além de obter melhorias na elaboração do escopo. Segundo Fairley (1994) muitos projetos de software não são entregues dentro do prazo acordado e dentro do custo planejado por não possuírem um modelo eficaz de gestão de risco.

No sentido de sistematizar o processo de desenvolvimento do aplicativo proposto neste estudo, foi utilizado o modelo clássico do ciclo de vida de desenvolvimento de software (Figura 1) de Royce citado por Forsberg, Mooz e Cotterman (2005), considerado como um 
modelo espiral composto por sete fases são: (a) Requisitos do sistema: representa o levantamento detalhado das necessidades dos usuários; (b) Requisitos de software: são as funcionalidades desejadas do aplicativo; (c) Designer preliminar: modelo inicial do aplicativo quanto a sua interface homem máquina; (d) Designer detalhado: modelo funcional do aplicativo; (e) Depuração de código: nesta fase feitos testes com a finalidade de encontrar erros lógicos no desenvolvimento; (f) Teste e pré-operação: nesta fase são efetuadas entradas de dados para validar suas saídas; (g) Operação e manutenção: esta fase representa o uso do aplicativo pelos usuários em seu cotidiano.

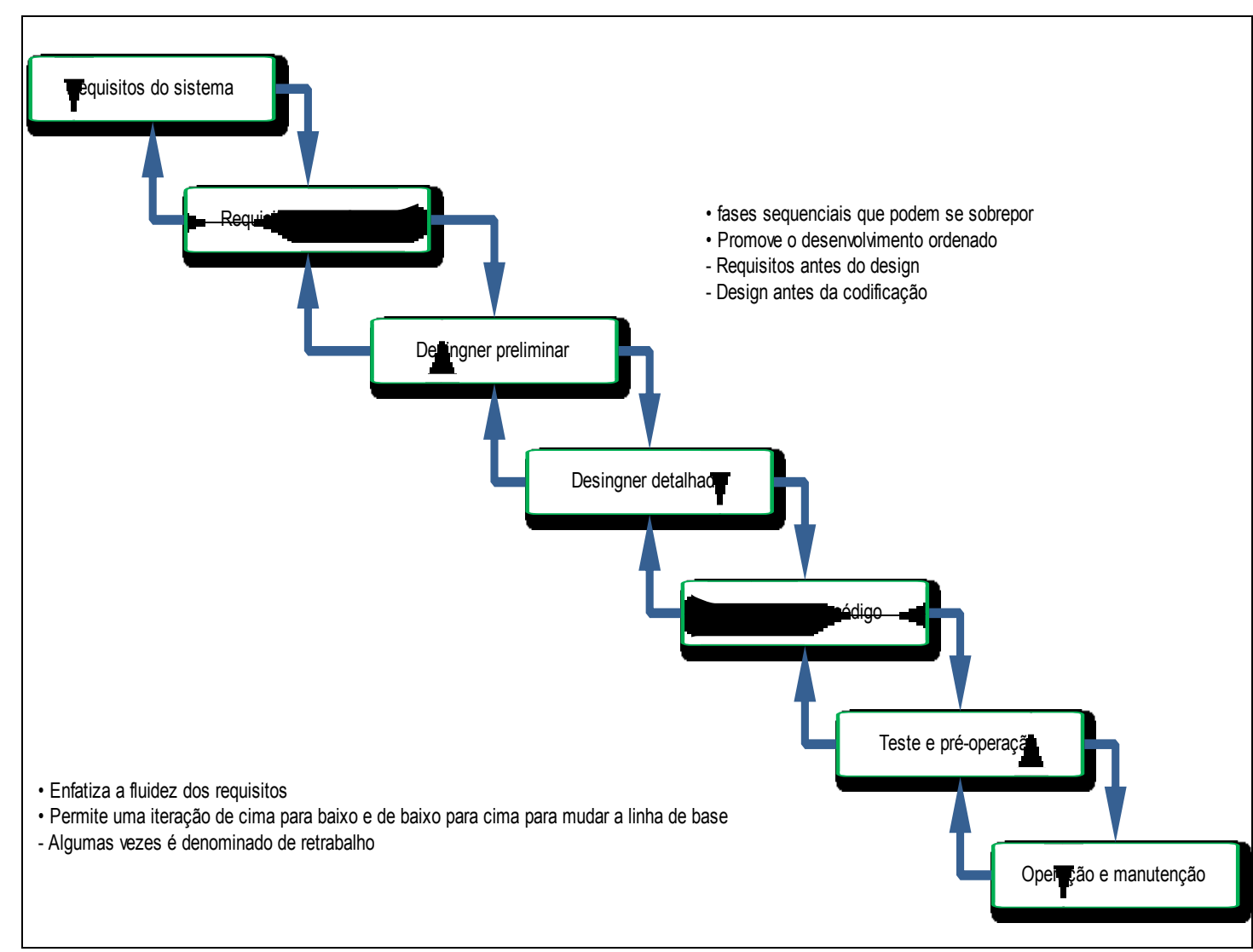

Figura 1 Ciclo de vida clássico do desenvolvimento de software. Fonte: Adaptado de Forsberg, Mooz e Cotterman (2005)

De acordo com Pressman (2006), o processo de desenvolvimento do software exige a confecção de documentos que servem de suporte ao levantamento e definição de requisitos e funcionalidades específicas do cliente ao qual devem ser implantadas ao novo software que está sendo desenvolvido. Com o escopo de todas as funcionalidades do software definido (fases de planejamento e análise), o centro da tomada de decisões se volta aos processos relacionados na fase de projetos. Em contraste com manufatura, os projetos de 
desenvolvimento de software são elaborados de forma mais integrada, em ciclos e fases mutuamente dependentes e sujeitas a verificações. Estes compreendem a integração de partes, peças e componentes destinados à construção de subsistemas e sistemas que irão compor o produto final - software definitivo.

\section{METODOLOGIA}

O método da Pesquisa-ação foi escolhido como estratégia de pesquisa para a realização do presente trabalho. Nesse sentido, foram escolhidos os princípios metodológicos que pautam esta pesquisa, os quais se baseiam nos estudos de Thiollent (1997), em particular quando o autor estabelece os princípios metodológicos gerais para pesquisa-ação inserida no contexto das organizações. Thiollent (1997) trata como organização qualquer entidade que tenha grupos sociais e suas atividades são estruturadas em processos com objetivos definidos.

O conceito então da pesquisa-ação está relacionado a junção de pesquisa e ação em um processo, onde os atores implicados participam junto com os pesquisadores, buscando de forma interativa esclarecer a realidade em que estão inseridos, identificando problemas coletivos, buscando e experimentando soluções em situação real. Desta forma, ao mesmo tempo, acontece produção e uso do conhecimento (THIOLLENT, 1997, 2008; MARTINS E THEÓPHILO, 2009).

Adicionalmente, Franco (2014) destaca a importância da pesquisa-ação como meio de promover conhecimentos partilhados, ressaltando a importância das espirais cíclicas como um fundamento essencial para estruturar os processos cognitivos os quais ocorrem durante a pesquisa.

A seguir será descrito o detalhamento da pesquisa-ação no desenvolvimento da pesquisa.

\subsection{FASES DA PESQUISA-AÇÃO}

De acordo com Thiollent (1997), a pesquisa-ação possui uma rotina composta por três ações principais que são: observar, para reunir informações e construir um cenário; pensar, para explorar, analisar e interpretar os fatos; e agir, implementando e avaliando as ações. Conforme Martins e Theóphilo (2009), a pesquisa-ação não é totalmente predefinida, porém, pode ser dividida em etapas: fase exploratória, fase da pesquisa aprofundada, fase de ação e 
fase de avaliação. O Quadro 1 apresenta um resumo das técnicas utilizadas no presente trabalho relacionadas com as fases da pesquisa-ação.

\begin{tabular}{|l|l|}
\hline \multicolumn{1}{|c|}{ FASE DO ESTUDO } & \multicolumn{1}{|c|}{ TÉCNICAS UTILIZADAS } \\
\hline Fase Exploratória: diagnóstico da situação atual. & $\begin{array}{l}\text { Entrevistas; observação, reuniões e análise } \\
\text { documental. }\end{array}$ \\
\hline $\begin{array}{l}\text { Fase da Pesquisa Aprofundada: Levantamento de requisitos. } \\
\text { Proposição do Sistema (aplicativo) de Acompanhamento. }\end{array}$ & Análise documental. \\
\hline $\begin{array}{l}\text { Fase de ação: Desenvolvimento e implementação do Sistema } \\
\text { (aplicativo) de Acompanhamento. }\end{array}$ & Reuniões, observação e análise documental. \\
\hline $\begin{array}{l}\text { Fase de Avaliação: Validação prática do Sistema (aplicativo) } \\
\text { de Acompanhamento. Observação do funcionamento para } \\
\text { ajustes e otimização. }\end{array}$ & $\begin{array}{l}\text { Observação e reuniões com usuários do } \\
\text { sistema. }\end{array}$ \\
\hline
\end{tabular}

Quadro 1 Ferramentas e técnicas aplicadas para desenvolver as atividades propostas.

Fonte: Elaborado pelos autores

Para o desenvolvimento do aplicativo de acompanhamento proposto foi necessário verificar a calibração de suas estimativas com base em dados anteriores. Neste sentido, foram escolhidos de maneira aleatória três Programas de Pós-graduação Stricto Sensu da área de Administração dos grupos de notas 5, 4 e 3, os quais participaram da avaliação trienal 20072009. Após a entrada de dados destes Programas foi possível verificar a eficiência do aplicativo comparando as notas obtidas pelos Programas com a nota gerada.

\subsection{DETALHAMENTO DA PESQUISA-AÇÃO NO DESENVOLVIMENTO DA PESQUISA}

A Figura 2 representa o detalhamento da Pesquisa-ação como estratégia de pesquisa no trabalho em questão, sendo que o modelo representado é cíclico dado à natureza da pesquisa. A seguir cada fase será descrita detalhadamente. 


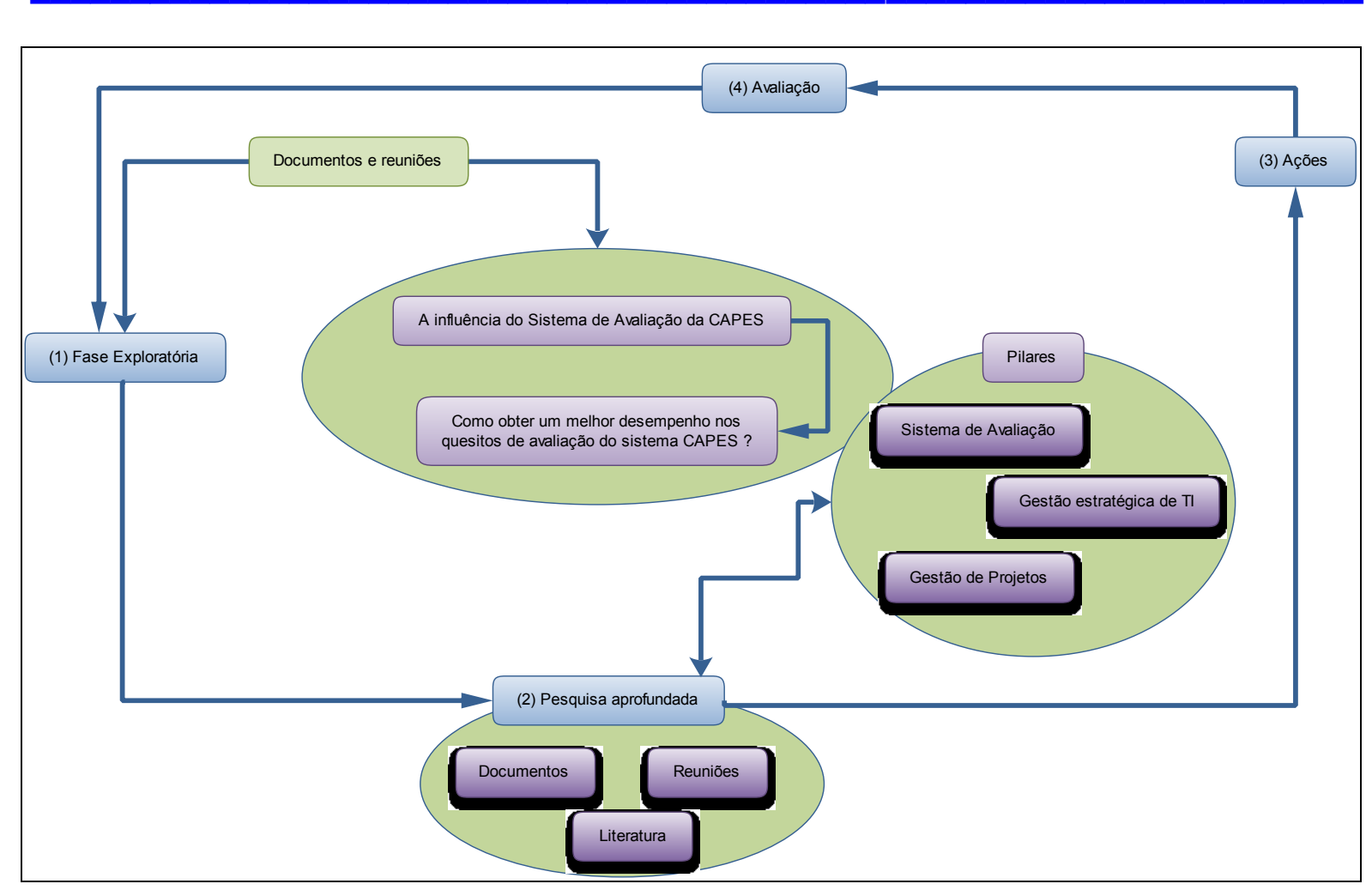

Figura 2 Modelo conceitual das relações da pesquisa-ação e a construção da pesquisa.

Fonte: Elaborado pelos autores.

a) Fase exploratória: Foi realizada a análise dos documentos disponíveis no sítio da CAPES, como caderno de indicadores de área, documento de área e relatórios de área. Nesta fase também foi realizada uma entrevista com o coordenador de um Programa de mestrado profissional em Administração que possui amplo conhecimento do sistema de avaliação da CAPES. O objetivo desta entrevista foi complementar a análise de dados secundários disponibilizados no sitio da CAPES com a opinião de indivíduos envolvidos no processo. $\mathrm{O}$ intuito foi compreender melhor o cenário no qual são planejadas e executadas as estratégias relacionadas à gestão da Pós-Graduação com base no sistema de avaliação da CAPES.

b) Fase da Pesquisa Aprofundada: Nesta fase foram coletados os dados referentes aos quesitos do sistema de avaliação da CAPES para os Programas de Pós-Graduação da área de Administração, foco da pesquisa, e elaborada a lógica para o desenvolvimento do modelo que dará suporte ao desenvolvimento do aplicativo. Desta forma foram elaboradas e propostas interfaces para o sistema de acompanhamento dos quesitos da CAPES, baseadas nos requisitos funcionais e interfaces de entrada e saída que deem subsídios às decisões a serem tomadas pelo gestor do Programa. Nesta fase foi utilizado os conceitos de gestão de projetos de TI com o objetivo de controlar as fases do projeto. O modelo utilizado é o proposto por 
Forsberg (2005), que ressalta que a melhor maneira de garantir o menor calendário e resultados de qualidade é por meio da aplicação de um ciclo de projeto, de maneira estratégica e tática gerido por pessoal qualificado e motivado.

c) Fase da ação: A fase da ação está associada a elaboração e implementação do aplicativo para os testes finais dos requisitos levantados. A escolha da linguagem de programação foi direcionada em função dos objetivos propostos, sendo que os testes de entrada de dados do aplicativo foram construídos de maneira a buscar possíveis erros de relações e interpretações dos requisitos levantados.

Neste sentido, os dados utilizados para tais testes são derivados do Relatório Coleta CAPES dos anos 2010, 2011 e 2012 de um Programa de mestrado profissional em Administração.

d) Fase da Avaliação: No caso deste estudo, o objetivo da fase de avaliação foi a validação prática do sistema desenvolvido na etapa anterior. Para tanto, foram necessários diversos debates e testes, para verificar a melhor forma para implementação, bem como treinamento dos atores envolvidos. Por meio dos dados do relatório Coleta CAPES (caderno de indicadores) dos anos de 2010, 2011 e 2012 de um Programa de mestrado profissional em Administração foram realizados testes com sistema consolidado para a obtenção de uma estimativa da nota da avaliação trienal do Programa.

\section{RESULTADOS E DISCUSSÃO}

Os resultados obtidos com o desenvolvimento da pesquisa são apresentados utilizando a mesma lógica do capítulo referente a Metodologia da Pesquisa, ou seja, por meio das fases da pesquisa-ação; fase exploratória, fase da pesquisa aprofundada, fase da ação e fase da avaliação.

\subsection{FASE EXPLORATÓRIA}

Por meio da análise dos documentos de área da CAPES, foi possível obter informações que foram utilizadas para elaborar uma planilha que representa os requisitos funcionais e parâmetros utilizados para o desenvolvimento do aplicativo de acompanhamento dos quesitos de avaliação proposto. A seguir destacam-se algumas informações relevantes sobre os quesistos de avaliação. 
a) Proposta do Programa - É uma descrição clara dos objetivos e proposta do programa, como também os objetivos na formação de seus mestres ou doutores, sendo subdivido em: a.1) Coerência, consistência, abrangência e atualização das áreas de concentração, linhas de pesquisa, projetos em andamento e proposta curricular; a.2) Planejamento do Programa com vistas a seu desenvolvimento futuro, contemplando os desafios internacionais da área na produção do conhecimento, seus propósitos na melhor formação de seus alunos, suas metas quanto à inserção social dos seus egressos, conforme os parâmetros da área; a.3) Infraestrutura para ensino, pesquisa e, se for o caso, extensão (CAPES, 2013).

b) Corpo Docente - Descreve a composição do corpo docente e seus respectivos relacionamentos com o programa de Pós-Graduação, sendo subdivido em: b.1) Perfil do corpo docente, consideradas titulação, diversificação na origem de formação, aprimoramento e experiência, e sua compatibilidade e adequação à Proposta do Programa; b.2) Adequação e dedicação dos docentes permanentes em relação às atividades de pesquisa e de formação do programa de Pós-Graduação; b.3) Distribuição das atividades de pesquisa e de formação entre os docentes do programa de Pós-Graduação; b.4) Contribuição dos docentes para atividades de ensino e/ou de pesquisa na graduação, com atenção tanto à repercussão que este item pode ter na formação de futuros ingressantes na programa de Pós-Graduação (CAPES, 2013).

c) Corpo Discente, Teses e Dissertações - Descreve as dissertações e os resultados obtidos pelos discentes do programa de Pós-Graduação, sendo subdivido em: c.1) Quantidade de teses e dissertações defendidas no período de avaliação, em relação ao corpo docente permanente e à dimensão do corpo discente; c.2) Distribuição das orientações das teses e dissertações defendidas no período de avaliação em relação aos docentes do programa de PósGraduação; c.3) Qualidade das Teses e Dissertações e da produção de discentes autores da Pós-Graduação e da graduação (no caso de IES com curso de graduação na área) na produção científica do programa, aferida por publicações e outros indicadores pertinentes à área; c.4) Eficiência do Programa na formação de mestres e doutores bolsistas: Tempo de formação de mestres e doutores e percentual de bolsistas titulados (CAPES, 2013).

d) Produção Intelectual - Objetiva a avaliação dos docentes e discentes em relação ao Qualis, sendo subdividido em: d.1) Publicações qualificadas do Programa por docente permanente; d.2) Distribuição de publicações qualificadas em relação ao corpo docente 
permanente do Programa; d.3) Produção técnica, patentes e outras produções consideradas relevantes (CAPES, 2013).

e) Inserção Social - Objetiva a avaliar o impacto social que o Programa de PósGraduação, traz na forma educacional, social, cultural, tecnológico/econômico, sendo subdividido em: e.1) Inserção e impacto regional e (ou) nacional do Programa; e.2) Integração e cooperação com outros programas e centros de pesquisa e desenvolvimento profissional relacionados à área de conhecimento do programa, com vistas ao desenvolvimento da pesquisa e da Pós-Graduação; e.3) Visibilidade ou transparência dada pelo Programa à sua atuação (CAPES, 2013).

Em relação a entrevista realizada com o coordenador de um Programa de mestrado profissional em Administração, foi possível identificar que os Programas necessitam realizar um acompanhamento sistematizado dos quesitos de avaliação da CAPES, sendo que este pode ser utilizado para a gestão estratégica do Programa de modo a obter os melhores conceitos. Esta consideração vai ao encontro das pesquisas realizadas por Maccari at. al. (2014).

\subsection{FASE DA PESQUISA APROFUNDADA}

De acordo com Martins e Theóphilo (2009), na fase da pesquisa aprofundada a direção da pesquisa é estabelecida em função dos resultados obtidos na fase anterior. Desta forma foi construída uma planilha com os parâmetros utilizados para a elaboração do aplicativo proposto, considerando-se os quesitos do sistema de avaliação da CAPES. Os resultados são discutidos a seguir com maior profundidade.

Como base para a elaboração do aplicativo de acompanhamento proposto neste trabalho, utilizou-se a planilha - documento orientador - estudado por Maccari et. al. (2009) que está apresentada na Tabela 2. Nesta planilha estão relacionados os itens que compõem cada critério do sistema de avaliação, de acordo com os critérios de avaliação da área de Administração, Ciências Contábeis e Turismo disponível no sitio da CAPES. A identificação da planilha atende assim a Etapa 3 do modelo de Forsberg (2005) utilizado nesta pesquisa.

Os conceitos Muito Bom, Bom, Regular, Fraco e Deficiente são atribuídos a cada um dos quesitos CAPES, objetivando a atribuição de uma nota final que pode ser 1, 2, 3, 4 e 5 . Ressalta-se que para os mestrados profissionais a nota máxima na avalição é 5. Cada um dos conceitos está relacionado a uma faixa, correspondente a um intervalo de variação. Para construção destes intervalos foram utilizados os dados do relatório Coleta CAPES um 


\section{LEVANTAMENTO DE REQUISITOS PARA A PROPOSTA DE UM APLICATIVO PARA ACOMPANHAMENTO DOS QUESITOS DO SISTEMA DE AVALIAÇÃO DA CAPES DOI: http://dx.doi.org/10.5007/1983-4535.2015v8n3p1}

Programa de mestrado profissional em Administração com nota 3 (nota anterior a sua primeira avaliação trienal). Desta forma foi possível calibrar cada um dos conceitos com uma respectiva faixa.

A faixa de qualificação dos conceitos utilizada no aplicativo refere-se aos conceitos, Muito Bom, Bom, Regular, Fraco e Deficiente, que representam respectivamente 100\%, 80\%, $60 \% 40 \%$ e $20 \%$ do quesito avaliado. Para a obtenção do valor correspondente à nota da avaliação trienal é utilizada a média referente a soma das notas de cada um dos anos do triênio.

Tabela 2 Parâmetros do sistema de Avaliação da CAPES para a área de Administração, Ciências Contábeis e Turismo.

\begin{tabular}{|c|c|c|c|c|c|c|c|}
\hline \multirow{3}{*}{ Quesitos } & \multicolumn{2}{|r|}{ Desdobramento do Quesito } & \multicolumn{5}{|c|}{ Parâmetros } \\
\hline & \multirow{2}{*}{ Peso } & \multirow{2}{*}{ Itens do Quesito } & $\begin{array}{l}\text { M. } \\
\text { Bom }\end{array}$ & Bom & Regular & Fraco & Def. \\
\hline & & & $100 \%$ & $80 \%$ & $60 \%$ & $40 \%$ & $20 \%$ \\
\hline \multirow{3}{*}{$\begin{array}{l}\text { Proposta } \\
\text { do } \\
\text { Programa }\end{array}$} & $50 \%$ & $\begin{array}{l}\text { 1.1. Coerência, consistência, } \\
\text { abrangência áreas de concentração, } \\
\text { linhas de pesquisa, projetos em } \\
\text { andamento e proposta curricular. }\end{array}$ & \multirow{3}{*}{\multicolumn{5}{|c|}{ AVALIAÇÃO QUALITATIVA }} \\
\hline & $30 \%$ & $\begin{array}{l}\text { 1.2. Planejamento contemplando os } \\
\text { desafios internacionais, formação de } \\
\text { seus alunos, metas quanto à inserção } \\
\text { social, egressos. }\end{array}$ & & & & & \\
\hline & $20 \%$ & $\begin{array}{l}\text { 1.3 Infraestrutura para ensino, } \\
\text { pesquisa e extensão. }\end{array}$ & & & & & \\
\hline \multirow{9}{*}{$\begin{array}{l}\text { Corpo } \\
\text { Docente } \\
20 \%\end{array}$} & \multirow{3}{*}{$15 \%$} & $\begin{array}{l}\text { 2.1 Perfil e experiência alinhada à } \\
\text { Proposta do Programa. }\end{array}$ & $\geq 90 \%$ & $80-90 \%$ & $70-79 \%$ & $60-69 \%$ & $\begin{array}{c}< \\
60 \%\end{array}$ \\
\hline & & $\begin{array}{l}2.2 \text { Diversificação de formação. } \\
\text { Proporção de docentes oriundos de } \\
\text { um mesmo PPG. }\end{array}$ & $<40 \%$ & $40-49 \%$ & $50-59 \%$ & $60-69 \%$ & $\begin{array}{c}< \\
60 \%\end{array}$ \\
\hline & & $\begin{array}{l}2.3 \text { Experiência na condução de } \\
\text { projetos de pesquisa. (Ao menos } 1 \\
\text { projeto no triênio). }\end{array}$ & $\geq 40 \%$ & $30-39 \%$ & $20-29 \%$ & $10-19 \%$ & $\begin{array}{c}< \\
10 \%\end{array}$ \\
\hline & \multirow{4}{*}{$30 \%$} & $\begin{array}{l}\text { 2.4 Adequação dimensão (Acad + } \\
\text { Prof) }\end{array}$ & $\leq 70 \%$ & $70-74 \%$ & $75-80 \%$ & $80-85 \%$ & $\begin{array}{c}> \\
85 \%\end{array}$ \\
\hline & & $\begin{array}{l}2.5 \text { Adequação dimensão (Acad + } \\
\text { Acad) }\end{array}$ & $\leq 50 \%$ & $51-54 \%$ & $55-59 \%$ & $60-64 \%$ & $\begin{array}{l}> \\
65 \% \\
\end{array}$ \\
\hline & & 2.6 Oscilação do Corpo Docente & $<25 \%$ & $25-34 \%$ & $35-44 \%$ & $45-54 \%$ & $\begin{array}{c}> \\
55 \%\end{array}$ \\
\hline & & $\begin{array}{l}\text { 2.7 Percentual de Docentes } \\
\text { Permanentes }\end{array}$ & $\geq 80 \%$ & $70-79 \%$ & $61-69 \%$ & $51-59 \%$ & $\begin{array}{c}< \\
50 \% \\
\end{array}$ \\
\hline & $30 \%$ & $\begin{array}{l}\text { 2.8. Distribuição das atividades de } \\
\text { pesquisa e de formação entre os } \\
\text { docentes do programa. }\end{array}$ & $\geq 90 \%$ & $\begin{array}{l}80- \\
89 \%\end{array}$ & $70-79 \%$ & $60-69 \%$ & $\begin{array}{c}< \\
60 \%\end{array}$ \\
\hline & $10 \%$ & $\begin{array}{l}\text { 2.9. Contribuição dos docentes para } \\
\text { atividades de ensino e/ou de pesquisa } \\
\text { na graduação, }\end{array}$ & $>90 \%$ & $80-89 \%$ & $70-79 \%$ & $60-69 \%$ & $\begin{array}{c}< \\
60 \%\end{array}$ \\
\hline
\end{tabular}




\section{LEVANTAMENTO DE REQUISITOS PARA A PROPOSTA DE UM APLICATIVO PARA \\ ACOMPANHAMENTO DOS QUESITOS DO SISTEMA DE AVALIAÇÃO DA CAPES \\ DOI: http://dx.doi.org/10.5007/1983-4535.2015v8n3p1}

\begin{tabular}{|c|c|c|c|c|c|c|c|c|}
\hline & $15 \%$ & \multicolumn{2}{|c|}{$\begin{array}{l}\text { 2.10. Participação do docente em } \\
\text { eventos alinhados à sua área de } \\
\text { atuação (mínimo } 1 \text { evento no triênio) }\end{array}$} & $\geq 80 \%$ & $70-79 \%$ & $61-69 \%$ & $51-59 \%$ & $\begin{array}{c}< \\
50 \%\end{array}$ \\
\hline \multirow{11}{*}{$\begin{array}{l}\text { Corpo } \\
\text { Discente } \\
35 \%\end{array}$} & \multirow{3}{*}{$10 \%$} & \multicolumn{2}{|c|}{$\begin{array}{l}3.1 . \% \text { de defesas mestrado em } \\
\text { relação ao total mestrandos }\end{array}$} & $\geq 40 \%$ & $35-39 \%$ & $30-34 \%$ & $25-29 \%$ & $\begin{array}{c}< \\
25 \% \\
\end{array}$ \\
\hline & & \multicolumn{2}{|c|}{$\begin{array}{l}\text { 3.2. \% de defesas doutorado em } \\
\text { relação ao total de doutorandos. }\end{array}$} & $\geq 22 \%$ & $20-21 \%$ & $18-19 \%$ & $\begin{array}{c}17- \\
17,9 \%\end{array}$ & $\begin{array}{c}< \\
17 \%\end{array}$ \\
\hline & & \multicolumn{2}{|c|}{$\begin{array}{l}\text { 3.3. Proporção de Permanentes com } 2 \\
\text { ou mais defesas no triênio (Para } \\
\text { PPGs com M e D foi calculada a } \\
\text { média) }\end{array}$} & $\geq 80 \%$ & $70-79 \%$ & $61-69 \%$ & $51-59 \%$ & $\begin{array}{c}< \\
50 \%\end{array}$ \\
\hline & $20 \%$ & \multicolumn{2}{|c|}{$\begin{array}{l}\text { 3.4.. Distribuição das orientações em } \\
\text { relação aos docentes do programa ( } 8 \text { - } \\
\text { 12). }\end{array}$} & $\leq 20 \%$ & $21-25 \%$ & $\begin{array}{l}<26- \\
30 \%\end{array}$ & $\begin{array}{l}<31- \\
35 \%\end{array}$ & $\stackrel{>}{35 \%}$ \\
\hline & \multirow{4}{*}{$50 \%$} & \multicolumn{2}{|c|}{$\begin{array}{l}\text { 3.5. Qualidade das T } \\
\text { examinadores externos }\end{array}$} & $\geq 95$ & $90-94 \%$ & $80-89 \%$ & $70-79 \%$ & $\begin{array}{c}< \\
70 \% \\
\end{array}$ \\
\hline & & \multicolumn{2}{|c|}{$\begin{array}{l}\text { 3.6.. Qualidade das } \mathrm{T} \text { e } \mathrm{D} . \% \text { de } \\
\text { trabalhos vinculados às linhas de } \\
\text { Pesquisa (Base nos Resumo) }\end{array}$} & $\geq 95$ & $90-94 \%$ & $80-89 \%$ & $70-79 \%$ & $\begin{array}{c}< \\
70 \%\end{array}$ \\
\hline & & \multicolumn{2}{|c|}{$\begin{array}{l}\text { 3.7. Qualidade das T e D. \% discentes } \\
\text { autores Mestrado (periódico/livro) }\end{array}$} & $\geq 35 \%$ & $20-34 \%$ & $10-19 \%$ & $5-9 \%$ & $<5 \%$ \\
\hline & & \multicolumn{2}{|c|}{$\begin{array}{l}\text { 3.8. Qualidade das T e D. \% discentes } \\
\text { autores Doutorado (periódico/livro) }\end{array}$} & $\geq 40 \%$ & $35-39 \%$ & $25-34 \%$ & $20-24 \%$ & $\begin{array}{c}< \\
20 \% \\
\end{array}$ \\
\hline & \multirow{3}{*}{$10 \%$} & \multicolumn{2}{|c|}{$\begin{array}{l}\text { 3.9. Eficiência do Programa na } \\
\text { formação de mestres (TMT) }\end{array}$} & $\leq 30$ & $30,1-31$ & $31,1-32$ & $32,1-33$ & $>33$ \\
\hline & & \multicolumn{2}{|c|}{$\begin{array}{l}\text { 3.10. Eficiência do Programa na } \\
\text { formação de doutores (TMT) }\end{array}$} & $\leq 54$ & $54,1-55$ & $55,1-56$ & $56,1-57$ & $>57$ \\
\hline & & \multicolumn{2}{|c|}{$\begin{array}{l}\text { 3.11. \% discentes e egressos com } \\
\text { publicação em eventos }\end{array}$} & $>40 \%$ & $30-39 \%$ & $20-29 \%$ & $10-19 \%$ & $\begin{array}{c}< \\
10 \% \\
\end{array}$ \\
\hline \multirow{5}{*}{$\begin{array}{l}\text { Produção } \\
\text { Intelectual } \\
35 \%\end{array}$} & $50 \%$ & \multicolumn{2}{|c|}{$\begin{array}{l}4.1 \text { Publicações qualificadas do } \\
\text { Programa por docente permanente }\end{array}$} & $\geq 150$ & $115-149$ & $90-114$ & $12-89$ & $<12$ \\
\hline & \multirow{2}{*}{$35 \%$} & \multicolumn{2}{|c|}{$\begin{array}{l}4.2 \text { Distribuição publicações } \\
\text { qualificadas em relação aos docentes } \\
\text { permanente ( } 150 \text { pontos) Métrica } 1 .\end{array}$} & $\geq 80 \%$ & $70-79 \%$ & $50-69 \%$ & $20-49 \%$ & $\begin{array}{c}< \\
20 \%\end{array}$ \\
\hline & & \multicolumn{2}{|c|}{$\begin{array}{l}\text { 4.3 Distribuição de publicações } \\
\text { qualificadas } \geq 80 \% \text { do NDP. Métrica } \\
\text { 2. (Métrica } 1 \times 2+\text { Métrica } 2 \times 1 \text { ) } / 3\end{array}$} & $>200$ & $121-200$ & $81-120$ & $41-80$ & $<40$ \\
\hline & \multirow[b]{2}{*}{$15 \%$} & \multirow{2}{*}{$\begin{array}{l}4.4 \\
\text { Produção } \\
\text { técnica/ } \\
\text { tecnológic } \\
\text { a }\end{array}$} & $\begin{array}{l}\text { Avaliação Qualitativa } \\
\text { (De acordo com } \\
\text { Documento de Área) }\end{array}$ & & \multicolumn{4}{|c|}{ AVALIAÇÃO QUALITATIVA } \\
\hline & & & $\begin{array}{l}\text { Avaliação Quantitativa- } \\
10 \text { produtos/atividades } \\
\text { (De acordo com o } \\
\text { relatório da avaliação } \\
\text { trienal) }\end{array}$ & $>50 \%$ & $40-49 \%$ & $30-39 \%$ & $20-29 \%$ & $\begin{array}{c}< \\
20 \%\end{array}$ \\
\hline \multirow{3}{*}{$\begin{array}{c}\text { Inserção } \\
\text { Social } \\
10 \%\end{array}$} & $50 \%$ & \multicolumn{2}{|c|}{$\begin{array}{l}5.1 \text { Inserção e impacto regional e/ou } \\
\text { nacional do programa (educacional, } \\
\text { social, cultural e tecnológico). }\end{array}$} & & \multirow{3}{*}{\multicolumn{4}{|c|}{ AVALIAÇÃO QUALITATIVA }} \\
\hline & $30 \%$ & \multicolumn{2}{|c|}{$\begin{array}{l}5.2 \text { Integração e cooperação com } \\
\text { outros programas. }\end{array}$} & & & & & \\
\hline & $20 \%$ & \multicolumn{2}{|c|}{$\begin{array}{l}5.3 \text { Visibilidade ou transparência do } \\
\text { programa. }\end{array}$} & & & & & \\
\hline
\end{tabular}

Fonte: Maccari (2008); CAPES (2013a; 2013d) 


\subsection{FASE DA AÇÃO}

Com base na fase anterior e na planilha construída (Tabela 2), foi elaborado o banco de dados do aplicativo, sendo que cada uma das opções do aplicativo representa um dos quesitos de avaliação da CAPES.

O cálculo final da nota da avaliação do Programa de Pós-Graduação em estudo é apresentado por ano (Tabela 3), representando uma simulação da nota. Destaca-se que cada quesito descrito na Tabela 3 possui os desdobramentos apresentados na Tabela 2 com sua respectiva avaliação.

Tabela 3 Tabela com os resultados consolidados da avaliação trienal de um Programa de Pós-Graduação Stricto Sensu da área de Administração.

\begin{tabular}{|c|c|c|c|c|c|c|}
\hline \multicolumn{2}{|c|}{ Quesitos } & M. Bom & Bom & Regular & Fraco & Deficiente \\
\hline & & $\mathbf{1 , 0 0}$ & $\mathbf{0 , 8 0}$ & $\mathbf{0 , 6 0}$ & $\mathbf{0 , 4 0}$ & $\mathbf{0 , 2 0}$ \\
\hline Proposta do Programa & $0,0 \%$ & $\mathrm{X}$ & & & & \\
\hline Corpo Docente & $20,0 \%$ & $\mathrm{X}$ & & & & \\
\hline $\begin{array}{c}\text { Corpo Discente, Teses e } \\
\text { Dissertações }\end{array}$ & $35,0 \%$ & & $\mathrm{X}$ & & & \\
\hline Produção Intelectual & $35,0 \%$ & & $\mathrm{X}$ & & & \\
\hline Inserção Social & $10,0 \%$ & & & & $\mathrm{X}$ & \\
\hline Média Global & \multicolumn{7}{|l|}{ BOM } \\
\hline
\end{tabular}

Fonte: Elaborado pelo autor.

\subsection{FASE DA AVALIAÇÃO}

Esta etapa final do processo de pesquisa-ação apresenta dois objetivos principais: verificar os resultados das ações no contexto organizacional da pesquisa e suas consequências a curto e médio prazo e extrair ensinamentos que serão úteis para a continuidade e aplicação em estudos futuros.

Thiollent (2008) afirma que é desejável que ocorra um retorno da informação entre os participantes que conversaram, participaram, investigaram e agiram. Este retorno promove então a visão de conjunto. Martins e Theóphilo (2009) apontam que as ações implementadas são neste momento objeto de profunda avaliação acompanhada por grupos conjuntos e sintetizadas.

Por meio dos dados do relatório Coleta Capes dos anos de 2010, 2011 e 2012 do Programa de mestrado profissional em Administração em estudo, foram realizados testes com o aplicativo, para a logica estabelecida, com o objetivo de obter uma estimativa da nota da avaliação triênal do Programa. A Figura 3 apresenta uma das telas do aplicativo referente aos 
testes realizados. Para execução deste teste foi utilizado a ferramenta de teste do visual studio 2010 .

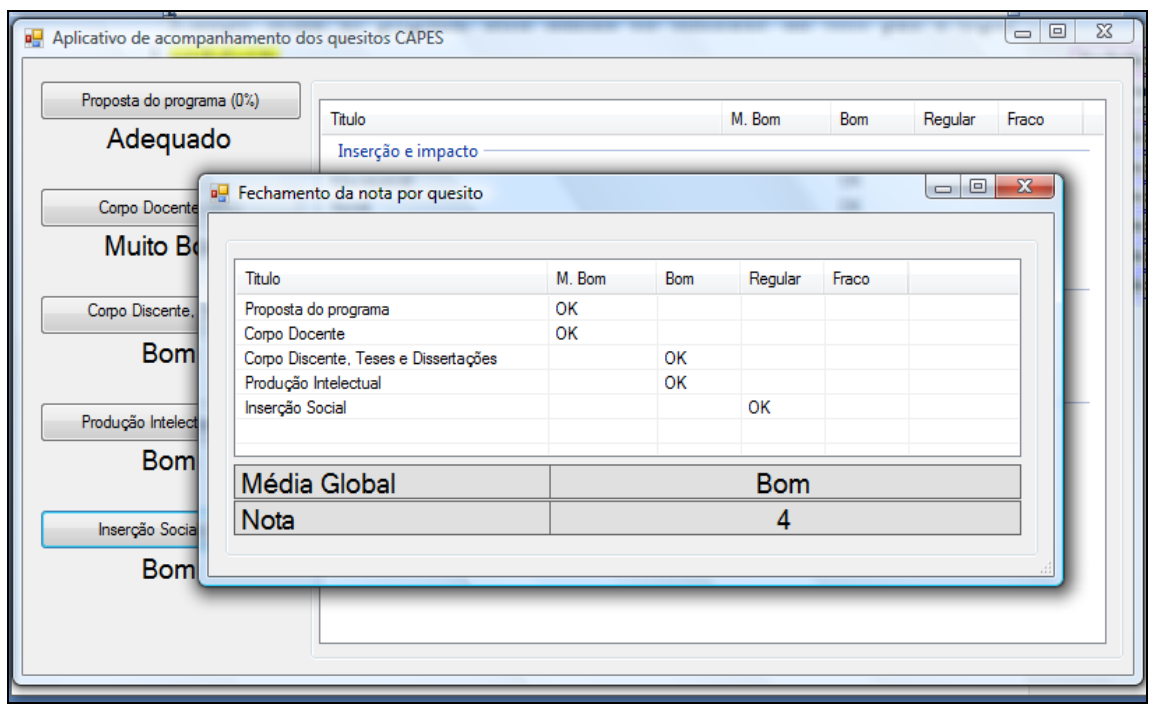

Figura 3 Janela do aplicativo referente ao calculo final da nota da avaliação do Programa testado.

\section{CONSIDERAÇÕES FINAIS}

Nas Instituições de Ensino Superior (IES) os departamentos de Pós-Graduação apresentam certo grau de independência em relação à graduação. No entanto, ainda que independente, a Pós-Graduação influencia fortemente a imagem e a visibilidade institucional. Desta maneira, cabe aos administradores e responsáveis pela gestão da Pós-Graduação construir e realizar estratégias eficientes e eficazes na condução destes setores (MACEDO, 2008).

Em relação ao estudo realizado, foi possível verificar o alinhamento das etapas da pesquisa-ação em conformidade com a gestão de projetos de TI no desenvolvimento do aplicativo proposto. Com relação a experiência de utilizar a pesquisa-ação no desenvolvimento de um aplicativo, observou-se que este foi um processo rico em geração de ideias e conceitos, contribuindo de maneira significativa para o seu desenvolvimento. Esta constatação está alinhada com a afirmação de Thiollent (2009), que retarta que construção de um projeto de pesquisa-ação em uma organização não é um procedimento de percurso único.

A validação do aplicativo em um Programa de Pós-Graduação da área de Administração mostrou que a lógica do mesmo é aplicável. Verificou-se que a utilização do aplicativo contribuiu para o acompanhamento dos quesitos de avaliação da CAPES, de forma a melhorar a alocação de recursos durante o período de avaliação. Neste sentido, sua 
utilização como ferramenta para a tomada de decisão contribui significativamente com a gestão estratégica de Programas de Pós-Graduação Stricto Sensu.

Os dados de um Programa de mestrado profissional em Administração que compuseram o teste do aplicativo demostraram a lógica estabelecida no sistema, haja vista que o resultado simulado para o Programa foi o conceito 4. Na avaliação trienal que ocorreu em 2013 referente aos anos 2010-2012, a eficácia do sistema foi comprovada, pois o Programa em questão obteve a nota 4 na avaliação do Comitê de Avaliação da área de Administração, Ciências Contábeis e Turismo da CAPES.

\section{REFERENCIAS}

BALBACHEVSKY, E. A Pós-Graduação no Brasil: novos desafios para uma política bemsucedida. In: BROCK, C.; SCHWARTZMAN, S. Os desafios da educação no Brasil. Rio de Janeiro: Nova Fronteira, 2005.

BOMENY, H. Newton Sucupira e os rumos da educação superior. Brasília: Paralelo 15, CAPES, 2001.128p. (Série Paralelo15) disponível em http://www.cpdoc.fgv.br acessado em $15 / 07 / 2012$.

CAPES. Documento de Área 2013 - Administração, Ciências Contábeis e Turismo. Brasília, 2013a. Disponível em: $<$ http://www.avaliacaotrienal2013.capes.gov.br/documento-de-area-ecomissao>. Acesso em: 13 jul. 2014.

CAPES. Relatório da Avaliação Trienal - Administração, Ciências Contábeis e Turismo. Brasília, 2013b. Disponível em: < http://avaliacaotrienal2013.capes.gov.br/relatorios-deavaliacao>. Acesso em: 13 jul. 2014.

, Plano Nacional de Pós-Graduação (Pnpg) 2011-2020. Volumes 1 e 2. Brasília, dezembro de 2010.

, Historia e Missão - Disponível em http://www.capes.gov.br/sobre-a-capes/historia-emissao? format=pdf acessado em 15/03/2012, 2012a.

, Avaliação da Pós-Graduação - Disponível em http://www.capes.gov.br/avaliacao/ avaliacao-da-pos-graduacao?format=pdf acessado em 15/09/2012. 2012 b.

Critérios APCNs - Disponível em http://www.capes.gov.br/avaliacao/avaliacao-dapos-graduacao? format $=$ pdf acessado em 15/09/2012. 2012 c.

, Sistema de Avaliação por fichas - Disponível em http://www.capes.gov.br/ avaliacao/sistema-de-ficha-de-avaliacao acessado em 15/09/2012. 2012 d. 
, Cadernos indicadores - Disponível em

http://conteudoweb.capes.gov.br/conteudoweb/ CadernoAvaliacaoServlet acessado em 15/09/2012. 2012e.

, Mestrados recomendados - Disponível em

http://conteudoweb.capes.gov.br/conteudoweb/ProjetoRelacaoCursosServlet?acao=pesquisar AreaAvaliacao acessado em 20/09/2014a

DAVENPORT, T. H.; PRUSAK, L. Ecologia da informação. São Paulo: Futura, 1998.

EARL, M. J. Experiences in strategic information system planning. MIS Quartely. v. 17, no 1, p. 1024, Mar. 1993.

FLORES, L.C.S. O Processo de informatização no Centro de Educação Superior de Ciências Sociais Aplicadas na Universidade do Vale do Itajaí. 1999. 135 p. Dissertação (Mestrado em Administração) - Universidade Federal de Santa Catarina, 1999.

FORSBERG, K.; MOOZ, H.; COTTERMAN, H.. Visualizing Project Management: Models and Frameworks for Mastering Complex Systems. Wiley, 2005.

FRANCO, M. A. S. Pesquisa-Ação: a Produção Partilhada de Conhecimento. UNOPAR Científica Ciências Humanas e Educação, v. 11, n. 1, 2014.

GATTI, B.; ANDRÉ, Marli; FÁVERO, Osmar e CANDU, Vera Maria F. O modelo de avaliação da CAPES. Revista Brasileira de Educação, jan-abr, n. 22, 2003.

HAMPTON, D. R. Management. New York: McGraw-Hill, 1986.

KAPLAN, ROBERT S.; NORTON, DAVID P. Alinhamento utilizando o Balanced Scorecard para criar sinergias corporativas. - Tradução Afonso Celso da Cunha Serra Rio de Janeiro: Elsevier, 2006.

(KERZNER, 2000; H. Applied Project Management - Best Practices on Implementation. New York: John Wiley \& Sons, 2000.

KUENZER, A. Z.; MORAES, M. C. T. Temas e tramas na Pós-Graduação em educação. Educ. Soc., Campinas, vol. 26, n. 93, p. 1341-1362, Set./Dez. 2005.

LEDERER, A.L. e SETHI, V. The Implementation of Strategic Information Systems Planning Methodologies. MIS Quarterly, 12, 3 (1988), 445-461.

LEDERER, A.L. MAHANEY, R.C. Using case tools in strategic information system planing. Information System Management Journal, p. 47-52, Fall 1996.

LUFTMAN, J. N.; BRIER, T. Achieving and sustaining business-IT alignment. California Management Review. California Management Review, Vol. 42 - Berkeley; 1999 
MACCARI, E. A.; ALESSIO, E. M; RODRIGUES, L. C.; QUONIAM, T. Sistema de Avaliação da Pós-Graduação da CAPES: Pesquisa-ação em um Programa de PósGraduação em Administração. X SEMEAD - Seminários em Administração FEA-USP, São Paulo, 2007.

MACCARI, E. A. Contribuições à Gestão dos Programas de Pós-Graduação Stricto Sensu em Administração no Brasil com Base nos Sistemas de Avaliação Norte Americano e Brasileiro, 2008 - Tese (Doutorado em Administração) - Universidade São Paulo, 2008.

MACCARI, E. A; ALMEIDA, M. I. R.; NISHIMURA, A. T.; RODRIGUES, L. C. A Gestão dos Programas de Pós-Graduação em Administração com Base no Sistema de Avaliação da CAPES. Revista de Gestão USP, São Paulo, v. 16, n. 4, p. 1-16, outubro-dezembro 2009.

MACCARI, E. A. et al. Proposta de um modelo de gestão de programas de Pós-Graduação na área de Administração a partir dos sistemas de avaliação do Brasil (CAPES) e dos Estados Unidos (AACSB). Revista de Administração, v. 49, n. 2, p. 369-383, 2014.

MACCARI, E. A.; LIMA, M. C.; RICCIO, E. L. . Uso do Sistema de Avaliação da CAPES por Programas de Pós-Graduação em Administração no Brasil. Revista de Ciências da Administração (CAD/UFSC), v. 11, p. 1-15, 2009a.

MACCARI, E. A.; ALMEIDA, Martinho Isnard Ribeiro de; NISHIMURA, A. T.; RODRIGUES, L. C.. A Gestão dos Programas de Pós-Graduação em Administração com Base no Sistema de Avaliação da CAPES. REGE. Revista de Gestão USP, v. 16, p. 1-16, $2009 b$.

MACEDO, D. L. Inteligência acadêmica como suporte à formulação de estratégias na gestão da Pós-Graduação stricto sensu em administração. São Paulo: Dissertação de mestrado do curso de Pós-Graduação em Administração da Universidade Nove de Julho, 2008.

MARTINS, G. A.; THEÓPHILO, C. R. Metodologia da investigação cientifica para ciências sociais aplicadas. São Paulo - Editora Atlas, 2009.

MORITZ, G. DE O., PEREIRA, M. F., MORITZ, M. O., \& MACCARI, E. A. (2013). A PósGraduação brasileira: evolução e principais desafios no ambiente de cenários prospectivos.

Future Studies Research Journal: Trends and Strategies, 5(2), 03-34.

MACHADO, L. D. Gestão Estratégica Para Instituições de Ensino Superior Privadas. Rio de Janeiro: Editora FGV, 2008.

MARCONI, M. A.; LAKATOS, E. M.. Técnicas de pesquisa: planejamento e execução de pesquisas, amostragens e técnicas de pesquisa, elaboração, análise e interpretação de dados. São Paulo: Atlas, 2006.

MARTINS, G. A.; THEÓPHILO, C. R. Metodologia da investigação cientifica para ciências sociais aplicadas. São Paulo - Editora Atlas, 2009. 
MARTINS, C. B., MACCARI, E. A., STOROPOLI, J. E., \& ANDRADE, R. O. B. de. (2013). Influência das estratégias e recursos para o desenvolvimento dos programas de PósGraduação da área de Administração, Ciências Contábeis e Turismo no período de 2001 a 2009. Revista Gestão Universitária na América Latina - GUAL, 6(3), 146-168.

MARTINS, G. A.; THEÓPHILO, C. R.. Metodologia da Investigação Científica para Ciências Sociais Aplicadas. 2a edição. São Paulo: Editora Atlas, 2009.

NEVES, R. B.; COSTA, H. G. Avaliação de Programas de Pós-Graduação: proposta baseada na integração ELECTRE TRI, SWOT e sistema CAPES. Sistemas \& Gestão, v. 1, n. 3, p. 276-298, setembro a dezembro de 2006.

OLIVEIRA, R. T. Q.; BOTELHO, E. N.; AMARAL, C. T. N.; ZOTES, L. P. Os Programas de Pós-Graduação Stricto Sensu no Contexto das Avaliações CAPES E CNPq. IV Congresso Nacional de Excelência em Gestão, Rio de Janeiro, 2008.

PARSON, G. L. Information technology: a new competitive weapon. Sloan Mannagement Review, v. 1, no 25, p. 3-14, fall 1983.


McGrawHill, 2006.

REZENDE, D. A. Engenharia de software e sistemas de informação. Rio de Janeiro: Brasport 1999.

REZENDE, D. A. Planejamento de Sistemas de Informação e Informática. São Paulo: Atlas 2011.

SAMPAIO, H. Ensino superior no Brasil - o setor privado. São Paulo: Fapesp/Hucitec, 2000.

STRINGER, E.T. Action research: A handbook for practitioners. Thousand Oaks, CA: Sage. 1996.

TACHIZAWA, T.; ANDRADE, R. O. B. Tecnologia da informação aplicada às instituições de ensino e às universidades corporativas. 2 ed. São Paulo: Atlas, 2003.

TURBAN, Efraim; RAINER, JR, R. Kelly; POTTER, Richard E. Introdução a Sistemas de Informação. Uma abordagem gerencial. Tradução Daniel Vieria - Rio de Janeiro: Elsevier, 2007.

THIOLLENT, Michel. Pesquisa-Ação nas Organizações. São Paulo: Atlas, 1997.

Metodologia da Pesquisa-ação. 16 ${ }^{\mathrm{a}}$ ed. São Paulo: Cortez (Coleção temas básicos de pesquisa-ação), 2008. 